\title{
REDES, CAMPOS Y MEDIACIONES: UNA APROXIMACIÓN SOCIOLÓGICA AL ARTE CONTEMPORÁNEO
}

\section{Fernando Golvano}

\begin{abstract}
RESUMEN
Este artículo aborda el carácter tautológico que es hegemónico en la creación artística contemporánea. Para el análisis de esa dinámica resultan pertinentes las aproximaciones sociológicas de Pierre Bordieu y de Raymonde Moulin, así como la perspectiva filosófica de Anne Cauquelin. Los conceptos de red, efecto bucle y campo artístico deben ser desarrollados para su aplicación en el ámbito del arte contemporáneo y para describir de modo más complejo las mediaciones que intervienen en su puesta en valor. Ahí tiene la sociología del arte un desafío sugerente para desarrollar un programa de investigación en diálogo con otras disciplinas científicas.
\end{abstract}

\section{INSTITUCIONES Y SISTEMA DEL ARTE}

Las Academias de Arte europeas, cuyo modelo se desarrolló en las ciudades italianas durante el siglo XVI, eran las que sancionaban el valor de las obras y de los artistas en los siglos XVII y XVIII. Su práctica cerrada y su escasa proyección pública se resquebrajó con el impulso democratizador dado por la Academia Francesa, que abrió libremente sus puertas a todo el mundo. A partir de ese momento y de la inauguración de los «salones» surge una nueva institución vinculada al sistema del arte: el público. Y en relación a él se desarrollará tanto la crítica de arte como el mercado. 
En esta nueva interacción exhibición/recepción pública de las obras de arte, los artistas — de modo especial, los que desafiaban los cánones académicos- depositaron sus esperanzas de legitimación y reconocimiento sin pasar necesariamente por la mediación académica. No obstante, ese optimismo y fascinación por la mediación crítica del público que visitaba los salones, devino en desconfianza para los artistas bohemios que pasaron a reivindicar la discriminación, donde la calidad recobraba sus derechos sobre la cantidad y el discernimiento sobre el espontáneo entusiasmo.

Con el desarrollo de la modernidad surgirán nuevas instituciones de mediación entre la obra y su recepción pública: la crítica de arte y la historia del arte. Por otro lado, la institucionalización de los salones constituyó uno de los primeros ejemplos de democratización de la cultura — debido al acceso de todas las clases sociales-, a la vez que emergieron nuevas prácticas estatales de intervención tutelada — qué o quiénes podrían exponer-. Esta dinámica de inclusión/exclusión provocó la creación de otros espacios alternativos, otros salones de los rechazados, de los independientes que se complementaron hacia el último tercio del siglo XIX, con la creación de galerías privadas.

Todo ello complejiza las agencias de mediación en el sistema del arte, en el que ni la academia, ni el museo, ni la galería, ni el marchante, ni el crítico de arte poseen el monopolio de la presentación pública o de la valoración crítica. También resulta necesario incluir a los poderes públicos que, a través de políticas culturales basadas en la democratización de la cultura, han alentado a las artes tanto al nivel de creación como al de difusión, exhibición y consumo.

Resulta significativa, por su escasez, la investigación sociológica que tenga en cuenta la complejidad creciente de las mediaciones que operan en el sistema del arte. Los ya clásicos estudios de A. Hauser $(1950)^{1}$ y de F. Antal $(1947)^{2}$ no superaban la reductora teoría del reflejo que primaba lo social sobre figurativo, utilizaban categorías esquemáticas e inmutables y privilegiaban el momento de la producción. Frente a este enfoque macrosociológico, Gombrich opondrá, en palabras de Castelnuovo (1988), un enfoque microsociológico: el análisis debe basarse en la «especificidad» del campo artístico, es decir, privilegia lo que ocurre dentro de una serie frente a los nexos con otras series — cultural, económica, política, etc.

Esa dicotomía micro/macrosociológica, será la que P. Francastel ${ }^{3}$ intentará

${ }^{1}$ Cfr. Historia Social de la Literatura y del Arte, 1985, Ed. Labor, Barcelona. Véase también su obra Sociología del Arte, 1975, Guadarrama, Madrid.

${ }^{2}$ Cfr. El mundo florentino y su ambiente social en los siglos XIV y XV, Guadarrama, Madrid, 1967.

3 Cfr. Sociología del Arte, 1984, Alianza Editorial, Madrid. Para Francastel: "La sociología del arte, implica, sí, un nuevo enfoque de una cierta categoría de objetos: los objetos figurativos y los monumentos, teniendo en cuenta que el artista representa una de las formas fundamentales de actividad del espíritu. Es, en consecuencia, a nivel de un análisis profundizado de las obras como únicamente se puede construir una sociología del arte. Nada serio puede hacerse si se toma el objeto de estos estudios como datos de la creación, en lugar de considerar las obras de arte como el producto de una actividad problemática, cuyas posibilidades técnicas, así como las capacidades de integración de sus valores abstractos, varían de acuerdo con cada uno de los ámbitos considerados» (Ibidem, p. 13). 
conciliar en su Sociología del arte (1970), reivindicando al mismo tiempo el carácter autónomo del arte y una función social de las formas artísticas. Pero esa antinomia se diluía cuando vinculaba la expresión artística a una visión del mundo. Una posición más prudente y mas pluricausal sobre las relaciones entre arte y sociedad es la postulada por F. Haskell (1963): «En ciertos momentos las conexiones entre condiciones económicas, políticas y un cierto estilo, me han parecido muy estrechas y en otros no he sido capaz de descubrir sino la lógica interna del desarrollo artístico, el capricho personal o la mano del azar» ${ }^{4}$.

La investigación sociológica en el ámbito de las artes y sus nexos con lo social ha tenido un débil desarrollo, a la vez que una destacable herencia de las Humanidades, hasta los años ochenta ${ }^{5}$. Algunos estudios recientes y significativos que abordan el sistema del arte y sus mediaciones son los de Becker (1988), E. Wallon (1991), R. Moulin (1986), A. Cauquelin (1992), N. Heinich (1989), N. García Canclini (1979) y P. Bourdieu (1988, 1995) ${ }^{6}$.

El concepto "contemporáneo» se utiliza de modo ambiguo en el arte: a veces designa un ámbito temporal que se inicia desde los postimpresionistas, otras la demarcación se aproxima a nuestro tiempo y se sitúa dentro del siglo $\mathrm{XX}$, en la emergencia de las vanguardias históricas. También hay quienes lo reservan sólo para el arte posterior a la II Guerra Mundial, y finalmente, algunos críticos adscriben la etiqueta de contemporáneo únicamente al arte nacido en los años sesenta. De modo que las fronteras entre arte moderno y arte contemporáneo están difusas tanto en su vertiente temporal como en la de su expresión artística. Pero, además, la esfera del arte se ha ido ampliando con nuevas formas de expresión que utilizan nuevas tecnologías.

A partir de 1968, los avatares del arte contemporáneo, su diversidad e innovación, han inducido nuevas propuestas de espacios de exhibición, estudio y conservación. Según Alonso (1993:148-149): las mutaciones que han afectado a esos espacios para arte contemporáneo, y en particular a los nuevos museos y centros de arte, han provocado cuatro enfoques, según Alonso (1993:148-149): "Con matices, podrían denominarse museos de la consagración histórica (muestran períodos y obras clave del arte contemporáneo universal),

${ }^{4}$ Citado por Castelnuovo (1988:68).

5 Para un análisis de la evolución de la sociología de las artes, véase V. ZOLBERG, «The arts and society in North America. Continuites and changes», P.-M. MENGER, «Les paradigmes de la sociologie de l'art", y A. Rodríguez Morató, "La sociología de las artes en el mundo», en I. Domínguez y A. Rodríguez Morató (1992), Arte, cultura y sociedad, AESCA, Barcelona.

${ }^{6}$ H. S. Becker (1988), Les mondes de l'art, Flammarion, París; E. Wallon (1991), L'artiste, le prince. Pouvoirs publics et creation, Museé de la Civilitation de Québec-PUG (Grenoble); R. Moulin (1986), «Le marché et le musée: la constitution des valeurs artistiques contemporaines», en Revue Française de Sociologie, XXVII, 3; N. HeINICH (1986), "La sociologie et les publics», en R. Moulin (dir.), Sociologie de l'art, La Documentation Française, París; N. GarCía CANCLINI (1979), La producción simbólica, teoría y método en sociología del arte, Siglo XXI, México; P. Bourdieu (1988), Cosas dichas, Gedisa, y (1995), Las reglas del arte. Génesis y estructura del campo literario, Anagrama, Barcelona. 
museos de la representación "cultural" (auténticos templos donde hoy se rinde culto al arte y a su boato, y donde destacan normalmente sus espectaculares ofertas de hipermercados culturales; museos —eventuales o no- de la significación experimental (innovadores e inusuales recintos museísticos, incisivos establecimientos de promoción del arte actual, que pueden presentarse como los ejemplos más alternativos o heterodoxos de la museografía contemporánea); y museos de la autolegitimación postilustrada (una nueva y esplendente estirpe de museos de arte concebidos más allá de los cánones y la herencia de la Ilustración; su flamante arquitectura, sus peculiares diseños de la colección y de la presentación de las obras demuestran, no obstante, una paradójica postura, propia sin duda de una situación de postindustrialismo "neoilustrado")».

La intervención creciente del Estado incorpora un mapa más complejo en la relación entre el mercado internacional del arte contemporáneo y el campo cultural en el que se incluyen las diversas agencias y agentes artísticos. Pero la crisis del Estado del Bienestar añade una nueva incertidumbre sobre la evolución del gasto público cultural y su reflejo en las políticas museísticas que favorecieron el furor museístico de las dos últimas décadas.

\section{LAS MUTACIONES DEL ARTE}

El arte contemporáneo en su permanente redefinición, deconstruye sistemáticamente los cánones y criterios tradicionales de belleza o las apuestas vanguardistas. Este proceso expansivo de las propias fronteras y diversificador de los valores que lo constituyen, conlleva un deriva hermenéutica en la medida que entraña a la vez el desplazamiento estético a la cuestión del sentido. Pero el arte contemporáneo no sólo provoca dificultad de comprensión en el público profano, sino también en el iniciado o especializado. Como advierte lúcidamente la socióloga Nathalie Heinich (1996: 203):

"Contrariamente, pues, a lo que podía pasar en los salones de pintura del siglo pasado con los detractores de la pintura moderna, ya no se trata de un problema de gusto, que entrañe litigios entre participantes de un mismo paradigma estético, sino de un problema de categorías cognitivas o de zócalos interpretativos, que entrañan diferencias entre categorías de público en lo sucesivo disjuntas, heterogéneas. Ya no se trata de discutir para saber si lo que se ve es bello o feo, si el artista tiene o no talento, si sabe o no pintar, sino que se trata de decidir si lo que ve es o no es arte, si su autor es o no un artista y, accesoriamente, cuáles son los criterios pertinentes en materia de arte. La cuestión de la belleza cede el paso a la cuestión de la autenticidad artística, la cual no se reduce ya, pues, a una querella de atribución (¿de qué mano es esta obra?) sino que se convierte, más generalmente, en un enfrentamiento sobre las fronteras del arte, 
incluso sobre los valores que conviene defender cuando la obra pone en escena la transgresión».

El relativismo estético dominante en las prácticas artísticas se desplaza también al momento de la recepción, de modo que estalla en una pluralidad de interpretaciones que impiden consensos o disensos generalizados. A partir de los años sesenta conviven estratos de aceptaciones o de rechazos. La escena del arte contemporáneo podríamos formularla como un espacio de competencias y sinergias, en el que posiciones distintas y coexistentes - que se corresponden con las diferentes prácticas y elecciones artísticas - se definen unas en relación a otras por proximidad, oposición o hibridación estética, o por su vinculación de ámbito desigual, y por la naturaleza de la red en la que se integran. No obstante, los artistas y las obras no fijan siempre una posición en ese espacio de redes, sino que fluctúan dinámicamente en función del capital cultural y del capital económico que vayan acumulando

Desde los años sesenta, el sistema del arte contemporáneo tiene una condición internacional. El vínculo entre "lo contemporáneo» y «lo internacional» constituye una de las mayores apuestas, en permanente revaluación, de la competición artística, tal y como lúcidamente lo ha descrito Raymonde Moulin (1995:206). De modo que la constitución de los valores artísticos contemporáneos es inseparable de la acción sinérgica establecida por un sistema de redes integradas por artistas, marchantes, críticos, conservadores, comisarios, museos, centros de arte, instituciones públicas y privadas... en una interacción compleja con un mercado ligado a la comunicación ${ }^{7}$ ¿Es posible modificar esos procesos de legitimación global y de jerarquización de los circuitos culturales? Probablemente, el desarrollo de los procesos globalizantes se complemente con la definición de otros de signo local o nacional, de manera que devenga una situación más compleja y flexible de polos múltiples, no focalizados exclusivamente en Europa occidental y en Estados Unidos ${ }^{8}$.

Pero, esa expansión de la red que permite nuevas entradas de artistas pertenecientes a otros ámbitos geográficos y culturales del resto del mundo, o de los lugares periféricos del Occidente hegemónico, conlleva la creación de nuevas semejanzas, diferencias y reestructuraciones. En las nuevas condiciones de la actual tendencia hacia, en palabras de Paúl Virilio, la "Glocalización» que designa «la paradoja aparente de una mezcla entre el tiempo local de una acti-

Cfr. R. Moulin (1986), «Le marché et le musée, la constitution des valeurs artistiques contemporaines», en Revue Française de Sociologie, XXVII-3, julio, París. Ese texto está incluido en una magnífica recopilación de ensayos suyos en R. Moulin (1995), De le valeur de l'arte, Flammarion, París, pp. 206-235.

${ }^{8}$ En ámbito español, la creación también está afectada por esa dinámica global: salvo un reducido grupo de artistas consagrados, la densa trama de artistas, galerías y centros de arte ocupa una posición subalterna en la red internacional mencionada. Tal situación se hace significativamente visible en los grandes eventos artísticos de carácter internacional: la Documenta, las bienales de Venecia, Lyon y Münster, o las diversas ferias internacionales. 
vidad aún precisamente situada y el tiempo global de la interactividad generalizada»" el mayor desafío para la práctica artística tal vez se desplace hacia su dual inscripción, local y global, lo cual conlleva la problematización de los modos de recepción e interpretación de las obras. Aquellas que sepan activar sentidos en un diálogo con los contextos específicos y con los contextos globalizados ocuparán una mejor posición en el escenario internacional.

Por otra parte, la creación actual sucede en un mundo cada vez más «sobreexpuesto». Volviendo a tomar prestadas palabras de Virilio, conviene reconocer la gestación de un «horizonte artificial» de una pantalla o monitor que es «susceptible de fijar permanentemente la preponderancia nueva de la perspectiva mediática sobre la inmediata del espacio ${ }^{10}$. Si a falta de un final de la historia estamos asistiendo a un final de la geografía, y a un incesante feed-back de los flujos comunicativos, sociales y económicos, el arte contemporáneo, sea cual sea su origen de creación, debe modular su relación con los diversos contextos. Una tentación, que algunos artistas no han sabido soslayar, es la búsqueda rápida de legitimación internacional a través de una práctica artística demasiado mimética de lo que se hacía en los centros que hegemonizan la escena mundial.

El relativismo estético, instituido como un valor central en la heterotópica escena artística, afecta también al protocolo de instalación y a las condiciones de interpretación y recepción del arte actual. Así las cosas, "arte contemporáneo» es una denominación polémica que zigzaguea entre categorías estéticas, museográficas, políticas, económicas y mediáticas.

\section{LA TEORÍA DE LOS CAMPOS DE PIERRE BOURDIEU}

En una conferencia en la École Nationale Supérieure des Arts Decoratifs, en 1980, Pierre Bourdieu reconocía que la sociología y el arte no se llevan bien $^{11}$. Repartía las culpas entre artistas y sociólogos y criticó dos ideas muy estudiadas sobre la sociología del arte y de la literatura, la primera es que la sociología explica el consumo cultural, pero no la producción. Sin embargo, para Bourdieu, «sólo se puede comprender el aspecto más específico de la producción en sí, es decir, la producción del valor (y de la creencia) si se toma en cuenta simultáneamente el espacio de los productores y el de los consumidores»" ${ }^{12}$.

La segunda idea afirma que la sociología - y su instrumento predilecto, la estadística - resta importancia a la creación artística, la aplasta, la nivela y

9 Paúl Virilio (1995), La vitesse de libération, Galilée, París, p. 165.

10 Paúl Virilio (1997), «¿Fin de la historia o fin de la geografía? Un mundo «sobre-expuesto», en Le Monde Diplomatique, 22-23, septiembre, p. 35.

${ }^{11}$ Pierre Bourdieu (1990), «¿Y quién creó a los creadores?», en Sociología y Cultura, Grijalbo, México, pp. 225-238.

12 Ibidem, p. 225. 
reduce. Según Bourdieu, como esa sociología reduccionista «olvida efectivamente lo esencial, es decir, ese universo que posee sus propias tradiciones, sus propias leyes de funcionamiento y reclutamiento, y, por ende, su propia historia, que es el universo de la producción artística. (...) La sociología de las obras culturales debe tomar como objeto el conjunto de las relaciones (las objetivas y las que se efectúan en forma de interacciones) entre el artista y los demás artistas, y, de manera más amplia, el conjunto de los agentes envueltos en la producción de la obra o, al menos, en la del valor social de la obra (los críticos, los directores de galerías, mecenas, etcétera)» ${ }^{13}$.

La aportación más significativa de Pierre Bourdieu a la sociología puede que sea su peculiar formulación teórica respecto a las cuestiones culturales y simbólicas. En el centro de muchas de sus investigacones está el abordaje crítico sobre cómo están estructuradas, en dual inscripción económica y simbólica, la reproducción y la diferenciación social. Así, una de sus aportaciones teóricas más sobresalientes es la teoría de los $\operatorname{campos}^{14}$ que le permite mediar entre los factores económicos y simbólicos, y entre lo individual y lo social. No niega la existencia de clases sociales, pero se opone a las tesis del marxismo reduccionista que afirma las determinaciones de lo económico sobre lo social.

$\mathrm{Su}$ esfuerzo de inducción teórica le conduce a trasladar hipótesis ancladas en el método comparativo, de un campo de lo social a otro (religioso, cultural, artístico, político). De este modo, para Bourdieu (1997:63):

«El propósito del análisis de las obras culturales consiste en la correspondencia entre dos estructuras homólogas, la estructura de las obras (es decir, de los géneros, pero también de las formas, de los estilos y de los temas, etc.) y la estructura del campo literario (o artístico, científico, jurídico, etc.), campo de fuerzas que indisolublemente es un campo de luchas. El motor del cambio de las obras culturales, lengua, arte, literatura, ciencia, etc., reside en las luchas cuyas sedes son los campos de producción correspondientes: estas luchas que pretenden conservar o transformar la relación de fuerzas instituida en el campo de producción tienen evidentemente el efecto de conservar o de transformar la esctructura del campo de las formas que son instrumentos y envites en estas luchas».

Aunque el campo es la estructura que determina la forma de las interacciones entre los artistas, y entre éstos y las agencias, no significa que su autonomía

13 Ibidem, p. 227.

${ }^{14}$ Desde sus primeros textos como el de "Campo intelectual y proyecto creador», en Jean Poullion y otros (1967), Problemas del estructuralismo (edición original en francés, en 1966), Siglo XXI, México, Bourdieu ha ido sistematizando sus teorías sobre el campo simbólico y cultural. Otras referencias significativas son: P. Bourdieu (1968), «Elementos d'une théorie sociologique de la preception artistique», en Review Internationale des Sciencies Sociales, XX, 4, pp. 4579; (1971), "Champ du pouvoir, champ intelectual et habitus de classe», en Scolies, 1, pp. 7-26; (1988), La distinción, Taurus, Madrid; (1990), Sociología y cultura, Grijalbo, México; (1995), Las reglas del arte, Anagrama, Barcelona; y (1997), Razones prácticas, Anagrama, Barcelona. 
sea completa. La autonomía relativa está también modulada por las condiciones sociales de producción del campo artístico que tiende a producir al artista como creador de objetos (fetiches) investidos como obras de arte. A través de la lucha de posiciones se temporaliza la historia del campo. Cada tendencia artística, escuela, corriente o nombre propio activa señas distintivas que tratan de señalar las propiedades atribuidas a un conjunto de obras o de productores. Estos instrumentos prácticos de clasificación que hacen las similitudes y las diferencias al nombrarlas, los producen los propios artistas o sus críticos titulares en la lucha por el reconocimiento y cumplen la función de signos de reconocimiento que distinguen a las galerías, a los grupos y a los pintores $\mathrm{y}$, también, los productos que fabrican o proponen (Bourdieu, 1995:237-238).

La contemporaneidad sucede en la lucha que sincroniza unos tiempos discordantes: los representados como vanguardia sólo tienen un público en el futuro, mientras que los conservadores sólo reconocen a sus contemporáneos en el pasado. Según Bourdieu (1995:240):

«El movimiento temporal que produce la aparición de un grupo capaz de hacer época imponiendo una posición avanzada se traduce por una traslación de la estructura del campo del presente, es decir, de las posiciones temporalmente jerarquizadas que se oponen en un campo dado, al así encontrarse cada una de las posiciones retrasada en un rango en la jerarquía temporal que también es al mismo tiempo una jerarquía social (las diagonales punteadas unen las posiciones estructuralmente equivalentes - por ejemplo, la vanguardia- en campos de épocas diferentes). La vanguardia se encuentra en todo momento separada por una generación artística (entendida como la separación entre dos modos de producción artística) de la vanguardia consagrada, a su vez también separada por otra generación artística de la vanguardia ya consagrada en el momento en el que a su vez ella entró en el campo».

El habitus es otro concepto ligado a la teoría del campo. Bourdieu lo define como un sistema de disposiciones durables y transferibles - estructuras estructuradas predispuestas a funcionar como estructuras estructurantes - que integran las experiencias pasadas y funcionan, en cada momento, como matriz estructurante de las percepciones y generadora de acciones (Bourdieu,1991:9394). Cabría aplicar ese concepto como principio generador y unificador que retraduce las características intrínsecas y relacionales de una posición en un estilo de vida y de prácticas, a un conjunto de artistas. Por ejemplo, las artistas que en el seno del body-art crean en clave feminista, definen un habitus generador de prácticas distintas y distintivas.

El habitus, como lo describe Bourdieu (1997:40), es esa especie de sentido práctico de lo que hay que hacer en una situación determinada. El desarrollo de muchas carreras artísticas, o de agentes actuantes en el campo artístico - marchantes, coleccionistas o directores de museos- dependen de cómo ges- 
tionan ese sentido práctico. La lógica del campo produce la illusio, esa adhesión colectiva al juego del arte, a sus propias reglas que no cesan de expandirse y reproducirse, y es a la vez causa y efecto de la existencia del juego. Estos postulados teóricos de Bourdieu cuestionan la ideología carismática y la fe en el creador, y desplazan la cuestión de la valoración y consagración de las obras y creadores a la noción de campo, a ese espacio de juego que se ha ido instituyendo progresivamente, y en el que se dan las luchas de posiciones y las condiciones de producción de la creencia.

\section{EL ARTE EN LA SOCIEDAD RED}

Manuel Castells (1997:88-89) recoge la noción de paradigma tecnológico, elaborada por Carlota Pérez, Christopher Freeman y Giovanni Dosi ${ }^{15}$ que, adaptando el análisis clásico de las revoluciones científicas de Kuhn, ayuda a organizar la esencia de la transformación tecnológica actual en su interacción con la economía y la sociedad.

Estas características descritas por Castells definen el nuevo orden social contemporáneo en el paradigma informacional y la sociedad red, provocan una perturbación sistémica que afecta a la dinámica del arte. En este contexto cobra actualidad el enfoque de redes que aplican al arte tanto Moulin (1995) como Cauquelin (1992).

Para Moulin (1986), las definiciones del arte contemporáneo se refieren bien a un criterio jurídico estrictamente cronológico, bien a un criterio de periodización histórica, bien a un criterio de categorización estética, o bien a la combinación de los dos últimos. Los años sesenta marcan una ruptura radical en la historia reciente del arte y coinciden con la internacionalización del campo artístico. El término "contemporáneo», en el sentido de los especialistas, es una etiqueta cuya obtención es una apuesta en la competición artística internacional, apuesta de las más ambiguas.

Así, en palabras de Moulin (1986), el mercado y el museo, sin que se sepa siempre cuál da el tono, contribuyen indisociablemente a la definición y a la jerarquización de los «valores» artísticos. El circuito cultural se impone al mercado como prueba de verdad. Las alzas rápidas que la publicidad, el marketing, los apasionamientos y las especulaciones provocan en el mercado no resisten mucho tiempo el reto de los profesionales del arte. Sin el veredicto autorizado de una fracción al menos de entre ellos, lo que se vende como arte no pertenece aún al universo del arte. El mercado del arte, incluso si tiene esta particularidad de poder ser manipulado simbólicamente a un nivel muy alto, queda como el lugar donde se operan las transacciones y donde se forman los precios.

15 Cfr. Carlota PÉrez (1983), "Structural change and the assimilation of new technologies in the economic and social systems», Futures 15, pp. 357-375; Giovanni Dosi y otros (1988), Technical Change and Economic Theory, Londres, Pinter. 
Por ahí está, de alguna manera, la prueba de realidad de las evaluaciones culturales y de los reconocimientos sociales operados fuera de él. Se ve, por otra parte, al Estado-Providencia intervenir para corregir la sanción, lastrada de realidad económica, del mercado, sea incrementando la parte de las inversiones públicas (compras y encargos), sea creando para los artistas «reconocidos» un mercado del empleo (y particularmente de la enseñanza) yuxtapuesto al mercado de las obras. A diferencia de los años 1870-1980, en los que el mercado del arte puso en práctica estrategias innovadoras que contestaron a la institución académica y aseguró, conjuntamente con una fracción de la crítica, el reconocimiento social y económico del arte independiente, el mercado actual obra/actúa en una interacción compleja con las instituciones culturales.

Otra socióloga francesa, Anne Cauquelin (1992), lleva más lejos la tesis de Moulin sobre el sistema del arte contemporáneo en una trama compleja de interacciones entre el mercado y las instituciones culturales. Cauquelin concibe ese sistema como una red ligada a la comunicación: «En términos de comunicación, la red es un sistema de relaciones multipolares, sobre el que se pueden conectar un número indefinido de entradas, en el que cada punto de la red general puede servir de punto de entrada de otras microrredes. Es decir, que el conjunto es extensible. En tal sistema lo que menos importa es la forma en la que se va a efectuar la entrada. Las informaciones que nos transmiten los diferentes medios de comunicación no tienen "autores". Las noticias provienen de redes interconectadas que se autoorganizan y se repercuten unas a otras. El autor es la metarred» (1992).

El efecto bucle es un concepto central para describir la dinámica de ese sistema de redes: la red de comunicación del arte contemporáneo se caracteriza por una imagen bucle (circularidad total del dispositivo), de manera que lo que se expone al público no son tanto obras singulares producidas por autores, sino imagen de la red misma. Cuando vemos una obra de arte contemporánea, vemos el arte contemporáneo en su conjunto. Se expone como totalidad y totalidad buclada. Esta tesis de A. Cauquelin se podría entender como la deriva, en la era de la comunicación, de la poética de conjunto que protegía y marcaba la presencia del artista y de la obra.

El consumidor está también en la red. En el sistema de red de comunicación en bucle, los destinatarios no son otros que los gestores de la red; es decir, que el fabricante-productor de la puesta en red de una obra la destina a sí mismo, y la consume después de haberla fabricado. El productor crea un artista no para venderlo sino para comprarlo él mismo y luego revendérselo (la obra/artista) a otros productores. El arte contemporáneo es su imagen, señala Cauquelin, y la realidad del arte se construye fuera de las cualidades propias de la obra, en la imagen que ella suscita a los circuitos de comunicación. En la diferencia entre mediaciones complicadas y mediaciones complejas (a través de la red) se aloja la diferencia que hay entre un mercado de consumo clásico y un mercado ligado a la comunicación.

Según el modelo que propone Cauquelin (1992), los elementos de la red son: 
A) Los productores. En el dominio artístico, los actores más activos son los que disponen de una gran cantidad de informaciones, provenientes del conjunto de la red, y esto lo más rápidamente posible. Estos actores privilegiados son: conservadores de grandes museos, importantes marchantes y galeristas, expertos, directores de fundaciones internacionales, los llamados "profesionales». Son ellos los primeros que obtienen y pasan la información: la de la cotización (el precio) y por consiguiente la del «valor» estético. Pero pasar la información, en una red de comunicación, es también fabricarla.

Cauquelin remarca los rasgos que se refieren implícitamente a la red comunicacional: el primero es la velocidad de transmisión de un punto al otro del mundo. El segundo es el avance del signo sobre la cosa: antes de haber sido expuesta, la obra del pintor/artista, o más bien su signo, circula ya en los circuitos de la red. El signo precede, pues, a aquello de lo que es signo. Así, de nuevo, la utilización de la red para la reventa entre los actores productivos. En fin, rasgo no despreciable, la puesta entre paréntesis del artista, el que «hace» el objeto-obra hace también el objeto de un intercambio de signos. Nada se refiere en este dipositivo a cualquier juicio estético por parte de los productores de valores.

B) Los niveles de producción. Existen redes de primer nivel y redes satélites. La red está estructurada por niveles jerarquizados e interconectados. Una de las características del poder-red es, en efecto, deportar/alejar el poder de decisión: ya no es central, no tiene lugar propio, no parte de un sujeto o de un grupo de sujetos para transmitirse a las periferias; una institución, incluso localizada y centralizada, no tiene poder más que en la medida en que es capaz de estar presente a la vez en toda la red.

C) El encargo. El encargo de obras proviene frecuentemente de instituciones como museos o departamentos de arte contemporáneo, fondos regionales de arte contemporáneo, de tamaño y modalidades diversas. Siendo así que estas instituciones tienen como función designar al público lo que es arte contemporáneo, son actores importantes en la red. Ésta no toma en cuenta el contenido de las transmisiones, sino solamente el hecho de la circulación de la información. Si hay muchas especificidades en la constitución de las redes parciales, no son especificidades de los contenidos, sino de su extensión. Así, el encargo no puede constituirse en red desconectada de las redes de profesionales y marchantes del primer tipo, pues es el mismo flujo de información el que les alimenta.

D) Los auxiliares de la producción. Los productores difunden sus informaciones a través de una red en la que se entremezclan la prensa especializada - portavoces, agencias, periodistas y críticos de arte, ligados a las galerías o a los museos-, los expertos y los comisarios de exposiciones, especie de escenógrafos para la visualización de las obras.

Así, puede decirse que en un sistema de comunicación en el que triunfa la red, sobrevienen efectos paradójicos: el profesionalismo, subrayado por todos los análisis críticos, corresponde a una especialización: la producción de arte 
corresponde a los grandes marchantes y coleccionistas —especialistas de la información y de la visualización - pero al mismo tiempo, en el interior de esta esfera de profesionalismo, los roles no están individualizados: un conservador de museo que muestra arte contemporáneo puede también escribir (prefacios de catálogos), puede ostentar la función de comisario de una exposición, puede incluso gestionar - cambiar o comprar obras y hacer subir la cotización, como todo buen especulador-, de manera que se coloque en el mercado internacional. El crítico, por su parte, puede muy bien no escribir, sino servir de introductor de las obras que ha elegido ante las galerías o los coleccionistas de su red. Puede también ser comisario de exposiciones o tomar el papel de experto adjunto a un museo de arte contemporáneo.

E) Los «artistas-creadores». En principio, y no sin contradicciones, la obra y el artista serán «tratados» por la red comunicacional a la vez como elemento constitutivo (sin ellos, la red no tendría razón de ser) y como un producto de la red (sin la red, ni la obra ni el artista tienen existencia visible). Son las nociones-principios de la comunicación — bucle, saturación y nominaciónlas que darán cuenta de su estatus contemporáneo.

En una sociedad de comunicación, la actividad más buscada, la más demandada, y quizá la única que conviene perfectamente a la circulación de informaciones sin contenidos específicos, capaces por lo mismo de asegurar el funcionamiento de las redes en tanto que puras redes, es la actividad de creación artística. La evidencia del sistema mismo se asegura, con un doble beneficio, según Cauquelin (1992): un beneficio ético que es: la igualdad de todos los intervinientes designados como creadores; y otro beneficio, éste de carácter político: que resulta de que el arte, al internacionalizárse, se convierte en el signo de una voluntad de agrupación, de consenso, de la cual los regímenes políticos no pueden sustraerse. La imagen simbólica de una nación se halla con este imperativo. De ahí las tomas de posición de un «Estado cultural».

Aun reconociendo la agudeza de las tesis de Cauquelin y la importancia de las redes en la valorización de las obras y los artistas, lo más problemático quizá sea la subestimación de otras variables que también intervienen en el sistema del arte como las elecciones personales o la inefable mano del azar. Con todo, lo más significativo es esa red compleja de sinergias entre museos, centros de arte, marchantes, galeristas, críticos, curators, instituciones públicas, fundaciones... que viene a ser otra forma de llamar a las musas enredadas. El propio Bonito Oliva, uno de los críticos-comisario de exposiciones con más vínculos y mejor posicionados en la red internacional del arte contemporáneo, reconoce que «el sistema del arte que incluye a los artistas, a los críticos, a los galeristas, a los coleccionistas, a los museos, a los mass media y al público, se ha convertido en el cauce dentro del cual se desarrolla el proceso creativo. La identidad artística inicial de la obra adquiere una plusvalía cultural, un valor añadido que, de forma progresiva a partir de los sesenta, viene determinada por la contribución de los restantes sujetos del circuito internacional del arte» 
(B. Oliva, 1990). En un texto más reciente (1995) advierte del peligro de la obra que "puede convertirse en gadget cultural", debido al drama de su acelerada circulación. Y añade: «Ahora, en cambio, no existen modelos, poéticas de grupo que reproduzcan anclajes culturales colectivos. La obra se entrega a la fantasía de cada artista, sin garantía alguna de solidaridad, de pertenencia social e ideológica. La estabilidad de la poética de grupo está constituida por las móviles precariedades de un circuito internacional que deshace la idea de familia artística o nacional en favor de una presencia solitaria que lucha contra otras presencias del mismo modo solitarias». Éstos son algunos rasgos movedizos de la compleja escena de espacios sociales, campos, redes y mediaciones que constituyen ese ámbito de lo social que denominamos arte contemporáneo.

No era posible en este artículo describir en profundidad un programa de investigación teórica sobre el específico campo de producción cultural que constituye el arte contemporáneo. Tampoco hemos intentado presentar los rasgos particulares de algunas prácticas o subcampos como el arte conceptual, el minimal art, el body art, el performance, el land art, el pop art, el expresionismo abstracto, el arte objetual y otras derivas contemporáneas. Todo esto queda para futuros programas de investigación aplicada. Lo más sustantivo de estas notas ha sido el reconocimiento de que cualquier aproximación sociológica a esos ámbitos de lo social debe contar con las aportaciones complementarias de tres dinteles críticos insustituibles en la definición del marco teórico: Pierre Bourdieu, Raymonde Moulin y Anne Cauquelin.

\section{BIBLIOGRAFÍA}

Alonso, L. (1993): Museología, Ediciones Istmo, Madrid.

ARPAL, J. (1991): «La representación cultural en sociedad urbana: escenarios metropolitanos y lugar social del arte», en $Z E H A R$, n. ${ }^{\circ} 10$, junio, Donostia.

Bonito Oliva, A. (1990): El arte hacia el 2000, Akal, Madrid.

- (1995): «Teoría de los sistemas: el arte», en Ars Mediterranea 7, Barcelona, pp. 64-69.

Bourdieu, P. (1990): Sociología y cultura, Grijalbo, México.

- (1995): Las reglas del arte. Génesis y estructura del campo literario, Anagrama, Barcelona.

- (1997): Razones prácticas. Sobre la teoría de la acción, Anagrama, Barcelona.

Calvo Serraller, F. [ed.] (1993): Los espectáculos del arte, Tusquets, Barcelona.

Castelnuovo, E. (1988): Arte, industria y revolución, Península, Barcelona.

Cauquelin, A. (1992): L'Art contemporain, Presses Universitaires de France, París.

- (1996): Petit traité d'art contemporain, Seuil, París.

HeINICH, N. (1996): «L'art contemporain exposé aux rejets: contribution à une sociologie des valeurs», en Hermés 20, pp. 193-204.

Moulin, R. (1986): «Le marché et le musée, la constitution des valeurs artistiques contemporaines», en Revue Française de Sociologie, XXVII-3, julio, París.

- (1995): De le valeur de l'art, Flammarion, París.

REQUeNA, F. (1989): «El concepto de red social», en REIS 48, pp. 137-152, CIS, Madrid.

Wallon, E. (1991): «Introducción», en VV.AA.: L'artiste, le prince. Pouvoirs publics et créacion, Musée de la Civilisation de Québec et Presees Universitaires de Grenoble. 


\begin{abstract}
This paper tackles the tautological nature which is hegemonic in contemporary artistic creation. In order to analyse this dynamic, the author deems it appropriate to examine the sociological approximations of Pierre Bourdieu and Raymonde Moulin, and the philosophical perspective of Anne Cauquelin. The network, loop-effect and artistic-field concepts should be developed in order to apply the in the field of contemporary art and to describe in a more complex manner the mediations which come into play when appraising them. In this respect, the sociology of art faces the thought-provoking challenge of developing a research programme in a dialogue with other scientific disciplines.
\end{abstract}

